\title{
REVISTA
}

Revista Educación

ISSN: 0379-7082

ISSN: 2215-2644

revedu@gmail.com

Universidad de Costa Rica

Costa Rica

\section{Caracterización de las prácticas sociales asociadas a la génesis de la inducción matemática: Un estudio exploratorio en estudiantes de ingeniería}

Cruz Quiñones, María De los Ángeles; Portillo Lara, Héctor Jesús; Tchoshanov, Mourat; Flores García, Sergio; Ramírez Sandoval, Osiel

Caracterización de las prácticas sociales asociadas a la génesis de la inducción matemática: Un estudio

exploratorio en estudiantes de ingeniería

Revista Educación, vol. 43, núm. 2, 2019

Universidad de Costa Rica, Costa Rica

Disponible en: http://www.redalyc.org/articulo.oa?id=44058158009

DOI: https://doi.org/10.15517/revedu.v43i2.30493

Esta obra está bajo una Licencia Creative Commons Atribución-NoComercial-SinDerivar 3.0 Internacional. 


\title{
Caracterización de las prácticas sociales asociadas a la génesis de la inducción matemática: Un estudio exploratorio en estudiantes de ingeniería
}

\author{
Characterizing Social Practices Associated with the Origins of Mathematical Induction: An Exploratory Study \\ among Engineering Students
}

Maria De los Ángeles Cruz Quiñones

Universidad Autónoma de Ciudad Juárez, México

maria.cruz@uacj.mx

(D) http://orcid.org/0000-0003-2107-6568

Héctor Jesús Portillo Lara

Universidad Autónoma de Ciudad Juárez, México

hector.portillo@uacj.mx

(iD http://orcid.org/0000-0001-6446-5235

Mourat Tchoshanov

University of Texas at El Paso, Estados Unidos

mouratt@utep.edu

(D) http://orcid.org/0000-0002-2852-4311

Sergio Flores Garcia

Universidad Autónoma de Ciudad Juárez, México

seflores@uacj.mx

(iD) http://orcid.org/0000-0001-8083-503x

Osiel Ramirez Sandoval

Universidad Autónoma de Ciudad Juárez, México

osiel.ramirez@uacj.mx

(iD http://orcid.org/0000-0003-3376-8406

\author{
DOI: https://doi.org/10.15517/revedu.v43i2.30493 \\ Redalyc: http://www.redalyc.org/articulo.oa?id=44058158009
}

(D)

Recepción: 05 Septiembre 2017

Aprobación: 13 Mayo 2019

\section{Resumen:}

Este trabajo pretende caracterizar las prácticas sociales asociadas a la génesis de la inducción matemática para dar sentido o significado a la inducción matemática. Primeramente, se hizo una revisión bibliográfica en torno a la problemática de la inducción matemática. Después, se efectuó un análisis histórico-epistemológico de la génesis de la inducción matemática comprendiendo dos épocas: la época de los siglos anteriores al siglo XVI y la de los siglos XVI y XVII. Basándonos en la aproximación teórica de la socioepistemología se identificó la práctica social asociada a la inducción matemática: la demostración. Considerando esta práctica, se analizó la existencia de elementos útiles para lograr un rediseño del discurso matemático escolar considerando al estudiantado de ingeniería (varias). En esta exploración cuatro estudiantes y dos docentes participaron. A través de un cuestionario exploramos las dificultades que el alumnado encuentra al resolver problemas de inducción matemática. Se concluye que la inducción matemática es un método de demostración importante en las matemáticas, pero debido a la práctica social de la demostración asociada a su génesis se infiere que su resignificación es muy compleja e inapropiada para llevarla al aula de estudiantes de ingeniería. Por ello, se recomienda un análisis histórico-epistemológico del siglo XVIII a la fecha para considerar el rediseño del discurso escolar.

Palabras ClaVE: Socioepistemología, Inducción matemática, Práctica Social, Fenómeno didáctico, Epistemología.

\section{Abstract:}


In an effort to understand the social practices and origin of Mathematical Induction, we conducted both a literature review on the teaching of Mathematical Induction and a historical-epistemological analysis of the subject dating back to two periods: prior to the $16^{\text {th }}$ century and during the $16^{\text {th }}-17^{\text {th }}$ centuries. The socioepistemological theoretical approach used to determine social practice for mathematical induction is the "proof". We also considered useful elements necessary to redesign mathematics school discourses specifically for engineering students. Four students and two instructors were asked to complete a survey about problemsolving difficulties that students struggled with. We conclude that although Mathematical Induction is an important method of proof in Mathematics, the origins of the social practice of the "proof" infers that its resignification is too complex and inappropriate for engineering students. A historical-epistemological analysis from the $18^{\text {th }}$ century to the present is recommended in order to redesign the educational discourse associated with this subject.

KEYWORDS: Socioepistemology, Mathematical Induction, Social Practice, Didactic Phenomenon, Epistemology.

\section{INTRODUCCIÓN}

La inducción matemática es un método de demostración complejo y que requiere un razonamiento inductivo. Este método consiste en asumir que $P(a)$ es verdadero donde $a$ es un entero fijo (caso particular). Se asume también que para cualquier entero $k$ mayor o igual que a, $P(k)$ también es verdadero. Entonces hay que probar que la suposición $P(k+1)$ es verdadera también. Este método ha sido parte del currículo escolar de los programas de ingeniería. Según Castro, Cañadas y Molina (2010) el razonamiento inductivo es un generador de conocimiento gracias a la generalización que parte de hechos abstractos, descubre patrones y constituye nuevo conocimiento. Sin embargo, existe un fenómeno didáctico asociado a la inducción matemática identificado en investigaciones en los últimos 30 años (Ernest, 1984; Andrew, 2007; Avital y Libeskind, 1978).

Los problemas asociados a la enseñanza y el aprendizaje de la inducción matemática tienen diferente naturaleza: cognitiva, didáctica y epistemológica (Andrew, 2007; Ernest, 1982,1984; Ron y Dreyfus, 2004). Estos son abordados en los antecedentes. Estos incluyen dos partes: una se centra en las dificultades en torno a la enseñanza y aprendizaje de la inducción matemática. La segunda parte es un análisis históricoepistemológico de la inducción matemática. Donde se identificaron las prácticas de referencia asociadas a la inducción matemática, así como la práctica social que regula estas prácticas. Todos estos elementos constituyen la construcción social de la inducción matemática.

La aproximación teórica utilizada en esta investigación es la socioepistemología. Ella tiene un papel fundamental en la matemática educativa. A través de la socioepistemología se pueden estudiar muchos objetos matemáticos desde un punto de vista social y con ello identificar prácticas que ayudan a la resignificación de un objeto matemático (Buendía, 2006; Montiel, 2005). Con ella se buscará una posible resignificación de la inducción matemática en el discurso matemático escolar para estudiantes de ingeniería de sistemas digitales, sistemas computacionales, eléctrica, manufactura, civil, biomédica, aeronáutica, mecatrónica, industrial y de sistemas.

Las preguntas de investigación de este trabajo son: ¿cuáles son las prácticas sociales que caracterizan el origen de la inducción matemática?; y, ¿una vez caracterizadas las prácticas sociales, existen elementos útiles para el rediseño del discurso matemático escolar? Por lo tanto, el objetivo de esta investigación es caracterizar las prácticas sociales asociadas a la génesis de la inducción matemática a través de un análisis histórico-epistemológico. Además de complementar el análisis con un estudio exploratorio de 4 estudiantes y 2 docentes. Este para ver si las prácticas sociales identificadas son compartidas por el estudiantado de ingenierías como industrial, sistemas computacionales y digitales. 


\section{Antecedentes}

Esta sección se divide en dos partes: la primera parte presenta la problemática en torno a la inducción matemática y la segunda parte es un análisis histórico-epistemológico de la inducción matemática. Por toda la problemática que trae consigo la inducción matemática es catalogada como un fenómeno didáctico. Existen investigaciones que reconocen la problemática encontrada al momento de utilizar inducción matemática (Ernest, 1982,1984; Ron y Dreyfus, 2004). Clasificamos esta primera parte de la problemática en tres apartados de acuerdo a la naturaleza del problema. El primer apartado son las dificultades cognitivas y técnicas de las y los estudiantes, el segundo son las dificultades didácticas, y el tercero son las dificultades epistemológicas de la inducción matemática. La segunda parte es acerca del origen de la inducción matemática presentada cronológicamente.

\section{Problemática de la inducción matemática}

\section{Dificultades cognitivas y técnicas de las y los estudiantes}

Las primeras investigaciones desarrolladas, donde se enfoca en el fenómeno didáctico asociado a la inducción matemática desde la componente cognitiva, se efectuaron después de 1980 (Ernest, 1982,1984; Avital y Libeskind, 1978). Desde entonces se han investigado las dificultades que encuentran los alumnos/as al tratar de comprender la inducción matemática (Avital y Libeskind, 1978; Andrew, 2007). Avital y Libeskind (1978) mencionan que una dificultad presentada en el estudiantado es cuando ven es el paso inductivo de la inducción matemática, aquí la dificultad se centra en entender ese paso. Esta dificultad ocurre debido a que se presenta un obstáculo epistemológico al tratar de asimilar el paso inductivo, al estudiantado se le complica demostrar algo como verdadero cuando se basa en algo que él no sabe o no está seguro de que sea verdadero. Es decir, el estudiante debe asumir que es cierto para n, entonces al asumirlo hace que él crea que hay una inconsistencia, y de ahí el demostrar lo siguiente es muy complejo para él. Para el estudiante la concepción de este paso es como hacer un castillo de arena sobre el mar, porque siente que no tiene la fundamentación necesaria para continuar y si el maestro no hace un énfasis especial en este punto, el alumno no llegará a asimilarlo.

La siguiente lista contiene algunas dificultades cognitivas y técnicas identificadas en el trabajo de las y los estudiantes (Ernest, 1984).

- La ambigüedad de la palabra inducción.

- El paso donde tú asumes algo que tienes que probar y luego lo pruebas.

- La forma lógica del principio de la inducción matemática.

- El paso base o base de la inducción al darle un valor de P1 o con Pn0.

- El uso exclusivo de series de sumas.

- Números naturales.

- Diferentes formas equivalentes del principio de inducción matemática.

- Forma axiomática

- Recurrencia

- Principio de inducción matemática (PMI)

- La manipulación algebraica y de identidades.

- No tienen representación visual (Andrew, 2007). 
Durante este análisis se identificó que, cuando se les presenta a las y los estudiantes un problema de inducción matemática equívoca, quedan desconcertados y confundidos porque aparentemente el problema estaba resuelto correctamente pero después descubren que no fue así. Entonces este tipo de problemas puede ser conveniente o no dependiendo del propósito que se tenga al poner este tipo de problemas, por lo cual, es recomendable considerarlo detenidamente. Este fenómeno didáctico asociado a la inducción matemática ha sido detectado por algunos docentes e investigadores (Avital y Libeskind, 1978; Ernest, 1984). Por ello, esta investigación recolectó y analizó la información necesaria y pertinente para el análisis de este fenómeno didáctico desde un enfoque social.

Dificultades didácticas. Es importante reconocer las dificultades didácticas, pues estas influyen en el aprendizaje del estudiantado, es decir el profesorado sigue teniendo un papel fundamental en el aprendizaje del estudiante (Tchoshanov, Cruz, Huereca, Shakirova, Shakirova, y Ibragimova, 2017). Algunas investigaciones reconocen dificultades didácticas al impartir el tema del principio de inducción matemática y concluyen con sugerencias o recomendaciones para minimizar estas dificultades didácticas (Avital y Libeskind, 1978; Ernest, 1984). Por ejemplo, si el profesorado no enseña de manera adecuada algún tema puede hacer que el alumnado repudie el tema. Si el cuerpo docente no tiene una manera muy clara de cómo se utiliza este método de inducción, y de cuál es su funcionamiento, llevarlo al aula de la forma adecuada para que se dé el proceso de enseñanza-aprendizaje sería muy complejo. Sin embargo, hay docentes que tienen ese obstáculo epistemológico; y dan clases, e imparten inducción matemática, pero esto puede limitar en gran parte el aprendizaje de una parte del estudiantado. En las dificultades de tipo epistemológicas se puede observar claramente lo abstracto y difícil que es este objeto matemático. Ahora bien, puede que el profesorado tenga un buen dominio de la inducción matemática, sea conocedor de cómo utilizarlo y todo lo referente al método, pero eso no significa que lo vaya a enseñar de una manera en la que cada estudiante pueda comprenderlo o que sepa cómo llevarlo al aula para que el alumnado llegue a adquirir una mejor apreciación del método (Mason y Spence, 1999). El lenguaje utilizado al enseñar inducción matemática es fundamental (Avital y Libeskind, 1978). Es importante el cómo aproximarse a la demostración sin ser muy formal o riguroso, explicando y mostrando las diferentes formas del PMI (principio de inducción matemática) equivalentes, para utilizar el método de inducción matemática donde el estudiantado haga sus propias conjeturas y pueda llegar a definir o formular este método para llegar a un buen entendimiento de él (Avital y Libeskind, 1978).

La siguiente lista muestra algunas de las dificultades didácticas que se presentan cuando el profesorado aborda el tema de inducción matemática en la clase.

- Falta de dominio (Avital y Libeskind, 1978).

- Falta de didácticas o situaciones de aprendizaje (Avital y Libeskind, 1978).

- Diferentes formas del PMI (Ernest, 1984).

- Manera muy axiomática de presentarlo en clase (Ernest, 1984).

- Carencias conceptuales (malos ejercicios) (Movshovitz, 1993).

En algunas investigaciones (Ernest, 1984; Cusi y Malara, 2008; Ron y Dreyfus, 2004) se hacen sugerencias de cómo abordar a la inducción matemática, qué forma de presentarla es más entendible o sencilla, y en ellas los autores sugieren alguna analogía, (una de ellas es la del efecto dominó, la cual es muy interesante y detalla cada paso) y así, de esta manera, se dan sugerencias para minimizar las dificultades del estudiantado. En la siguiente lista se resumen algunas de esas sugerencias:

- Forma de presentarlo.

- El paso de $P(1)$ a $P(k)$ dar valores de $\mathrm{n}=1,2,3,4$, es decir $P(1), P(2), P(3), P(4)$ y con ello conjeture el $P(n)$ o $P(k)$.

- Ejemplos previamente seleccionados. 
- Propiedades de los números naturales y ver lógica matemática.

- Diversidad de ejercicios.

Dificultades epistemológicas. Estas son propias del conocimiento matemático en cuestión, algunas pueden suceder debido a la creación de la inducción matemática, otras por la representación de ella, otras por el uso y sus aplicaciones, y otras por la esencia de la inducción matemática es decir por lo que es, por su epistemología. Algunas de las dificultades epistemológicas surgen de su creación o generación de este objeto matemático como se verá en la sección de la inducción matemática en su contexto de origen.

Un resumen de algunas de las dificultades epistemológicas de la inducción matemática identificadas se da a continuación (Ernest, 1982, 1984).

- Axioma que define los números naturales

- Comunicación

- El lenguaje simbólico de la lógica

- El razonamiento deductivo

\section{La inducción matemática en su contexto de origen}

El acercamiento a la historia y la epistemología de la inducción matemática es con el enfoque de la matemática educativa. Esto para tratar de encontrar los fundamentos necesarios y propios de dicho objeto matemático, para que con ellos y el análisis histórico-epistemológico se pueda hacer una resignificación de la inducción matemática. Es decir, se buscarán los argumentos para darle un sentido o significado distinto y en ocasiones hasta contrastante de cierto concepto, y con ello llegar a desarrollar un rediseño del discurso matemático escolar para que se pueda dar una mejor aproximación al proceso de aprendizaje y enseñanza de la inducción matemática.

En análisis histórico se identificaron dos periodos: la época que comprende los siglos anteriores al siglo XVI y la época que comprende los siglos XVI y XVII. En estos periodos se encuentran las primeras evidencias de la inducción matemática. Para poder apreciarlas se tiene que hacer el análisis de ellos en su contexto de origen no se pueden analizar sin tomar en cuenta las situaciones y el modus vivendi que había en esos periodos.

Se elaboró este análisis histórico-epistemológico utilizando obras originales o trabajos especializados en procesos o conceptos relacionados con la inducción matemática, su epistemología y su historia. Todo lo anterior hecho con la finalidad de identificar cómo es que surge la inducción matemática, saber cuáles son los factores sociales o situaciones que influyeron para que se haya generado dicho conocimiento matemático.

El primer periodo inició aproximadamente 300 años a.e.c. y comprende trabajos de investigación ejecutados en distintas culturas como la de los árabes, griegos, etc., donde se observan indicios importantes de la inducción matemática hasta antes del siglo XVI. Los primeros indicios se encontraron en La obra Elementos de Geometría de Euclides (1991). Otros indicios fueron en trabajos de teoría de números de las comunidades griegas (Cajori, 1909, 1918). Como en ese tiempo se carecía de la notación de la edad moderna, no era fácil identificar la inducción matemática como se conoce actualmente. Después de estos trabajos no se encontraron indicios de la inducción matemática en algún trabajo durante siglos.

En el segundo periodo fue descubierta la evidencia más contundente de la inducción matemática. Se encontró la más importante prueba de la inducción matemática en un trabajo de un matemático italiano Francesco Maurolico (1494-1575) (Cajori, 1918; Ernest, 1982). En este periodo las comunidades europeas de matemáticos estaban todavía anonadadas por los geómetras griegos posteriores a Euclides. Una actividad que proliferaba en este periodo era la reconstrucción de trabajos o tratados griegos. También en estos siglos empezaba la corriente del álgebra en Europa, así como la geometría analítica de Fermat y Descartes. 
Se elaboró un estudio epistemológico de carácter histórico de la inducción matemática, donde se identificó la inducción matemática en los trabajos de grandes personajes (Napolitani, 2000; Pascal, 1995; Bussey, 1917). Francesco Maurolico plasmó la inducción matemática en su obra la arithmeticorum libri duo en el primer libro (Napolitani, 2000). La obra consiste en definiciones de números y sus proposiciones. Maurolico inicia su obra definiendo algunos números como los enteros, pares, impares, etc. (ver Figura 1). De ahí empieza a desarrollar proposiciones que manejan estos números y hacen sus demostraciones, en algunos casos utilizando proposiciones anteriores.

\begin{tabular}{|c|c|c|c|c|c|}
\hline Integers & Boen & Odd & Triangular & Square & N. P. A. L. \\
\hline 1 & 0 & 1 & 1 & 1 & 0 \\
\hline 2 & 2 & 3 & 3 & 4 & 2 \\
\hline 3 & 4 & 5 & 6 & 9 & 6 \\
\hline 4 & 6 & 7 & 10 & 16 & 12 \\
\hline 5 & 8 & 9 & 15 & 25 & 20 \\
\hline 6 & 10 & 11 & 21 & 36 & 30 \\
\hline 7 & 12 & 13 & 28 & 49 & 42 \\
\hline . & . & . & . & . & . \\
\hline . & . & - & . & - & . \\
\hline . & $\dot{F}$ & $\dot{0}$ & $\dot{T}$ & $\dot{s}$ & $\dot{L}$ \\
\hline$n$ & $E$ & 0 & $T$ & $S$ & $L$ \\
\hline
\end{tabular}

FIGURA 1

Numeraciones de Maurolico Bussey (1917, p.201)

En las proposiciones XIII y XV es donde Maurolico utilizó argumentos de la inducción matemática entre algunas otras (Napolitani, 2000; Bussey, 1917) (ver figura 2).

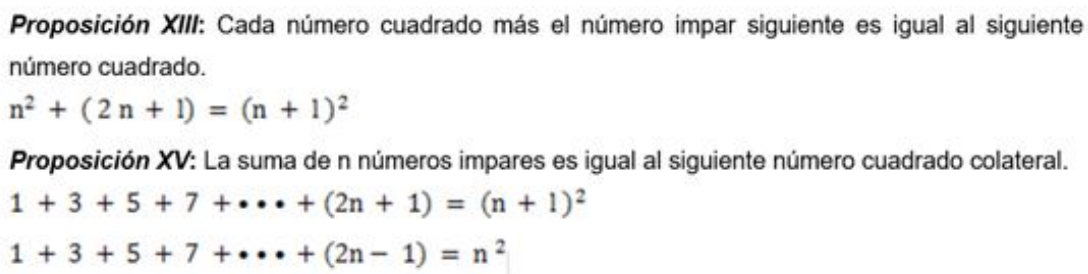

\section{FIGURA 2}

Proposiciones de Maurolico Fuente: Elaboración propia

Se analizaron las dos proposiciones mostradas en la Figura 2. Se observa claramente que la proposición XIII es utilizada como un lema. Esto provee el argumento de $\mathrm{n}$ a $\mathrm{n}+1$. Los primeros casos especiales son mencionados en la proposición XV. Con una notación más moderna y simbólica se puede tener más claro cómo emerge la inducción matemática en este trabajo de Maurolico (Napolitani, 2000), por ello se resume y ejemplifica en la Figura 3. 


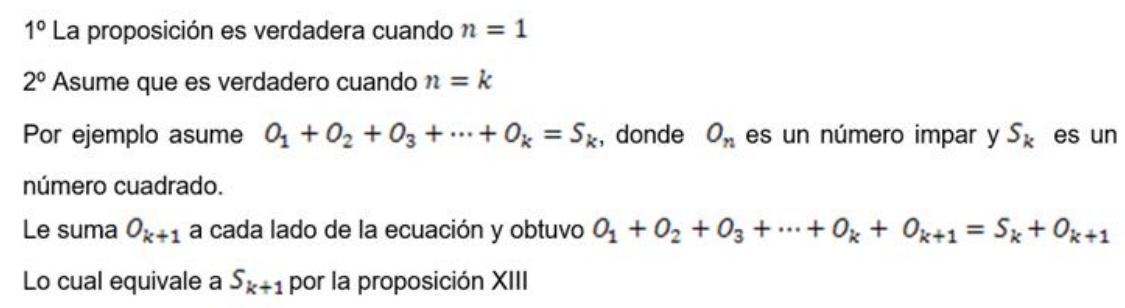

FIGURA 3

Inducción matemática de Maurolico

Bussey (1917, p. 212)

Otro personaje muy importante en el siglo XVII en la inducción matemática fue el francés Pierre de Fermat (1601-1655). Fue en un documento llamado Relation des decouvertes en la science des nombres donde se encontró un método de demostración que es llamado la descente infinite ou indefinite (Cajori 1909). Este es conocido actualmente como el método descendente y consiste básicamente en lo siguiente (ver Figura 4).

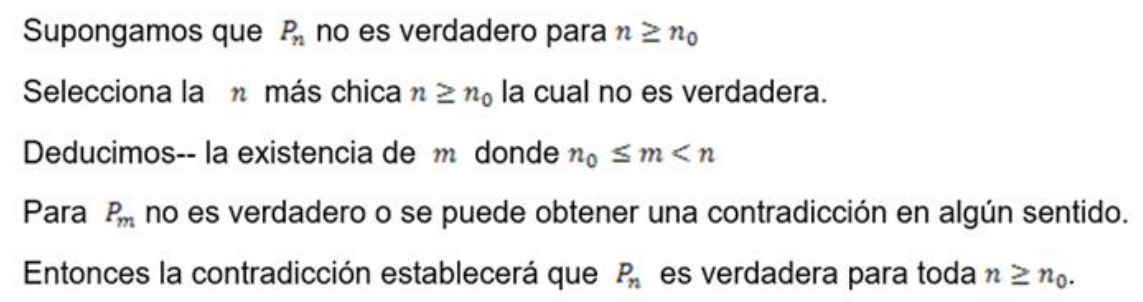

FIGURA 4

Inducción matemática de Fermat Ernest (1982, p.121)

La estrategia de Fermat fue para deducir la existencia de una secuencia infinita descendiente de unos números cuadrados de la forma negativa del teorema. Una secuencia de números naturales que está estrictamente descendiendo no puede existir, Fermat mostró que la forma negativa de un teorema lleva a una contradicción y de ahí que el teorema es verdadero (Ernest, 1982). La principal característica de este método es la suposición de que un conjunto no vacío de números naturales contiene al menos un elemento y esa característica es la que lo hace equivalente a la inducción matemática. Es decir, el método de Fermat consiste en asumir lo opuesto de lo que quiero probar y mostrar que la suposición conduce a una contradicción. Cualquier prueba de inducción matemática puede ser presentada en forma descendente.

El francés Blaise Pascal (1623-1662) empezó describiendo el triángulo aritmético, dando sus propiedades y componentes, así como sus 19 consecuencias (Pascal, 1995). Pero fue en la consecuencia décima segunda donde la formulación de la inducción matemática es evidente.

Consecuencia XII

En todo triángulo aritmético si se toman dos células contiguas sobre una misma base, la superior es a la inferior como la multitud de las células desde la superior hasta lo alto de la base es a la multitud de aquellas desde la inferior hasta abajo inclusive.

Si bien esta proposición tiene una infinidad de casos daré una demostración muy corta suponiendo 2 lemas.

El 1, que es evidente de suyo, que esta proporción se encuentra en la segunda base, pues es muy evidente que $\phi$ es a $\sigma$ como 1 al.

El 2, que si esta proporción se encuentra en una base cualquiera, se encontrará necesariamente en la siguiente (Pascal, 1995, p.60). 
Como se puede apreciar se utilizó un método de demostración que utiliza dos lemas los cuales son los que actualmente se conocen como paso base y paso inductivo. A lo largo del tratado del triángulo aritmético se pueden observar varias demostraciones de donde se puede extraer la expresión de la inducción matemática, una de ellas es la mencionada anteriormente, otra es en la proposición I del uso del triángulo aritmético para las combinaciones, entre otras.

En diferentes partes del mundo y diferentes personas utilizaron la inducción matemática, sin embargo, hay que considerar que la forma en la cual se identifica en esas investigaciones no es precisamente la misma que se tiene actualmente de la inducción matemática (Napolitani, 2000; Pascal, 1995; Bussey, 1917; Cajori, 1909, 1918). Esto es debido a que no se tenía un lenguaje tan simbólico como ahora. La notación utilizada anteriormente era diferente a la actual, sin embargo, se analizaron estos trabajos porque de alguna manera $u$ otra contienen alguna idea o sentido de la inducción matemática.

\section{Aproximación teórica}

\section{La construcción social de la inducción matemática}

La matemática se ha ido construyendo socialmente en la vida cotidiana de una forma particular para cada individuo, interactuando este con diferentes aspectos como su estilo de vida, sus necesidades, sus docentes, las escuelas, el conocimiento, etc. Para ser más específico se menciona el siguiente ejemplo: al inicio de la humanidad cuando las personas dejaban de ser nómadas y empezaban a convertirse en seres humanos sedentarios surgieron en ese momento muchas necesidades distintas entre ellos, una de esas necesidades fue la de medir su territorio para así poder conservarlo. Esto trajo consigo que se implementaran distintas formas de medición, como medición con partes del cuerpo, medición con pasos, etc., aquí es donde surge el saber matemático que ellos implementaron para satisfacer dicha necesidad. Así es como se construye la matemática y al querer llevarla al aula de una forma rigurosa y de una manera en la que el alumnado no le encuentra sentido, una manera muy diferente y en algunos casos hasta contrastante a la forma en que se construyó causa una infinidad de conflictos en el estudiantado. Por ello se deben hacer modificaciones para dar una resignificación al concepto matemático en cuestión y se llegue a dar una mejor apreciación de las matemáticas. Desde la perspectiva de la socioepistemología, investigar la generación del conocimiento de un objeto matemático, saber cómo surgió y se construyó, considerando el contexto social de su construcción, puede contribuir a adecuar las situaciones en clase para abordar dicho objeto matemático (Buendía, 2004). Aquí es donde esta investigación reconoce la necesidad que existe de elaborar estudios los cuales, necesariamente, articulan y reconocen la parte social, pues las dificultades que presenta el estudiantado no solo dependen de la falta de experiencia matemática o de habilidades, o de la idiosincrasia de su inmaduro pensamiento, sino de la naturaleza misma del concepto matemático y de la cultura en el marco que se desarrolló (Covián, 2005).

La socioepistemología es una aproximación teórica de la matemática educativa donde se busca la construcción social del conocimiento matemático por medio de un producto de factores socioculturales y epistemológicos (Buendia, 2006). Es decir, que al identificar y comprender la génesis de la inducción matemática, así como su evolución a lo largo de la historia, se llega a una aproximación socioepistemológica. El contexto sociocultural se refiere al aspecto social y cultural que acompañan la construcción de dicho conocimiento matemático.

La socioepistemología considera cuatro aspectos fundamentales: el cognitivo, el didáctico, el epistemológico y el social. Las interacciones entre estos cuatro aspectos facilitan que se dé un desarrollo del conocimiento matemático en las y los estudiantes (Montiel, 2005). Es decir, esta teoría considera al individuo como el aspecto cognitivo y por ende, se tiene el carácter social de lo cognitivo. En el aspecto didáctico se analizan todos los mecanismos de enseñanza, que por lo general son aquellos que los y las maestras utilizan 
en clase, los que están en los libros de texto, el programa de estudio, el discurso del docente que siempre es visto como algo dogmático, incuestionable, y todo ello es lo que estudia esta teoría desde un punto social (ver Figura 5). Además, se estudia el aspecto epistemológico donde el conocimiento es construido en un espacio o contexto sociocultural.

Una de las tareas de la socioepistemología consiste en la formulación de epistemologías de prácticas, las cuales permiten conformar bases de significados para el conocimiento y para su introducción significativa y articulada al sistema didáctico (Buendía y Cordero, 2005). Las prácticas sociales son las que producen el conocimiento matemático. Por ello la socioepistemología se enfoca en el rol de dichas prácticas y toma en cuenta los aspectos anteriormente mencionados. Las prácticas sociales son consideradas como normativas o reguladoras de la actividad humana, es decir las prácticas sociales son aquello que hace que los individuos o grupos hagan lo que hacen (Covián, 2005).

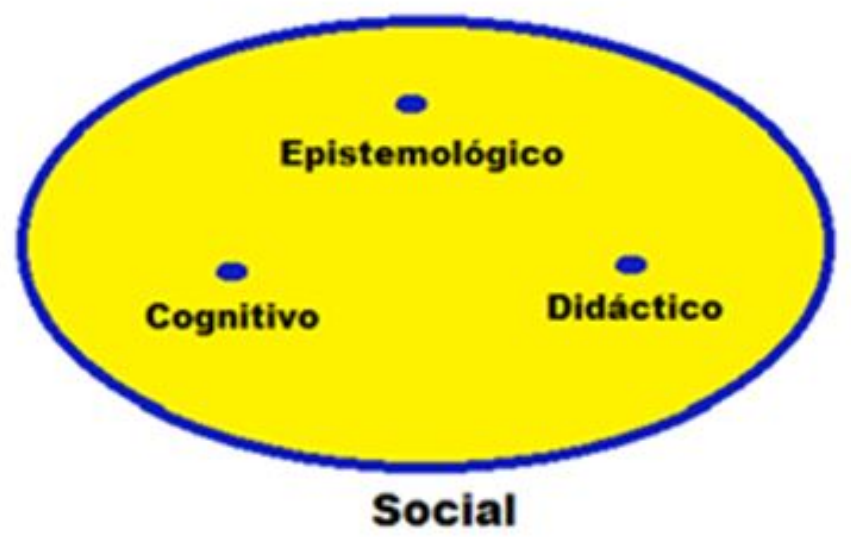

FIGURA 5

Las interacciones de los componentes de la socioepistemología Fuente: Elaboración propia

Según Montiel (2005), la epistemología de prácticas se conforma por tres componentes las prácticas sociales, de referencia y sus actividades. La práctica social regula las prácticas de referencia, las cuales consisten en un conjunto de actividades. Como se observa en la Figura 6, las tres están vinculadas para hacer la resignificación del conocimiento matemático en cuestión. Es decir, la práctica de referencia es la definición de una serie de actividades que distinguen a un grupo específico en un determinado contexto, estas actividades son ejecutadas por ese grupo y referencian esa época o contexto sociocultural. 


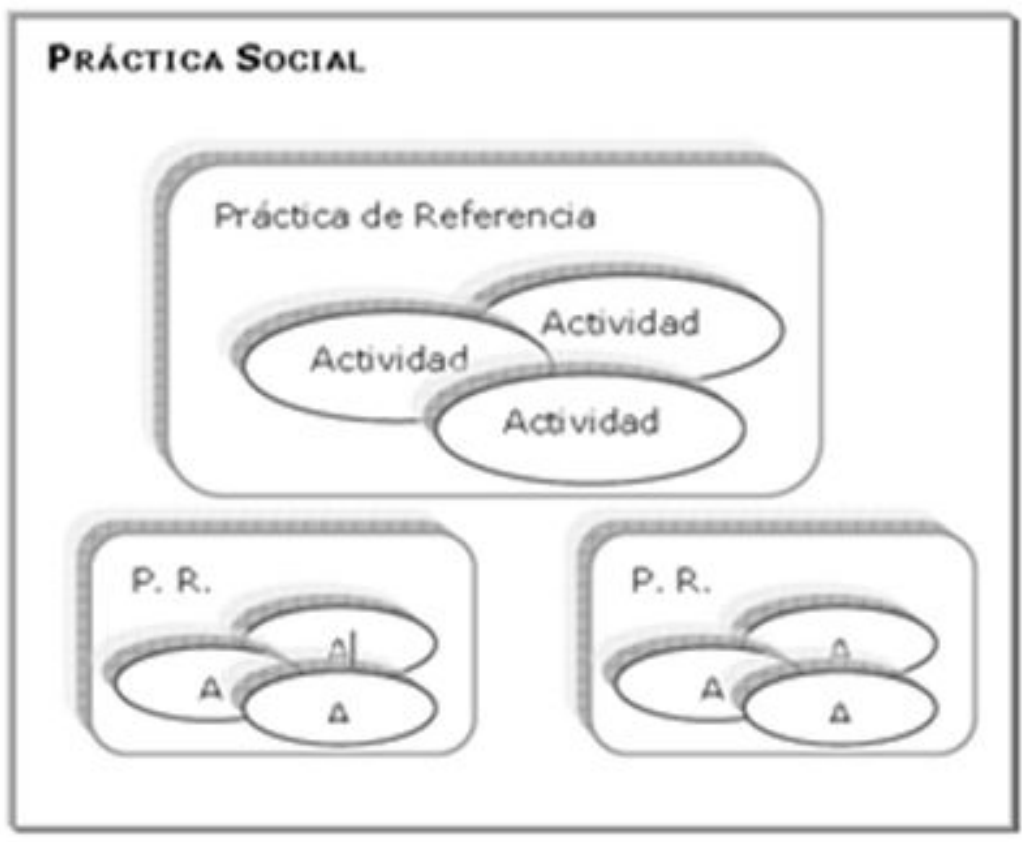

FIGURA 6

Modelo de práctica social de Montiel

Montiel (2005, p.101)

\section{Metodología}

La metodología utilizada en este trabajo es de naturaleza cualitativa. Este tipo de metodología consiste en un conjunto de prácticas interpretativas que permiten brindar una realidad a través de representaciones en forma de observaciones, cuestionarios abiertos, diálogos, entrevistas, video o audio grabaciones, y documentos (Sampieri, 2006). Se implementa esta metodología para encontrar el significado con el que la inducción matemática fue originada y explorar si existen elementos útiles para el rediseño del discurso matemático escolar para estudiantes de ingeniería.

El diseño metodológico de esta investigación parte del reconocimiento de un fenómeno didáctico. Se le conoce como fenómeno didáctico a las acciones erróneas o conflictivas (complejas, o difícil de entender) que ocurren constantemente en los ámbitos escolares, es decir se llevan a cabo de manera regular en distintas aulas y en diferentes lugares. Este reconocimiento se hizo en los antecedentes en la parte de la problemática en torno la inducción matemática.

Después se elaboró un análisis histórico-epistemológico presentado en los antecedentes. Esto se llevó a cabo para saber cómo se construyó, en qué contexto sociocultural, etc. Está es una estrategia de la metodología cualitativa, también conocida como investigación histórica tiene como propósito describir un evento del pasado a través de registros, documentos, historias orales, fotos, entre otros artefactos. Se analizaron las obras originales para investigar la inducción matemática, cuando no fue posible, se aproximó lo más que se pudo.

Basándose en el análisis, se desarrolló una epistemología de prácticas de carácter histórico-epistemológico que buscó identificar las prácticas que hacen que se genere o construya dicho conocimiento. Estas prácticas deben ser reinterpretadas para desarrollar e implementar un diseño o experimentación (situación), donde se va evidenciar el papel de las prácticas y dar una resignificación del conocimiento matemático, para llevar estos resignificados a los mecanismos de enseñanza y puedan ser implementados en el aula. 
La Figura 7 muestra el diseño metodológico llevado a cabo en esta investigación. Una vez identificadas las prácticas se buscan elementos útiles para un diseño en el aula de matemáticas. Para lograr esto, se elaboró un estudio exploratorio con estudiantes y docentes. Se aplicó un cuestionario a las y los estudiantes y se investigaron los documentos (notas de clase) que muestran cómo se abordó el tema de inducción matemática.

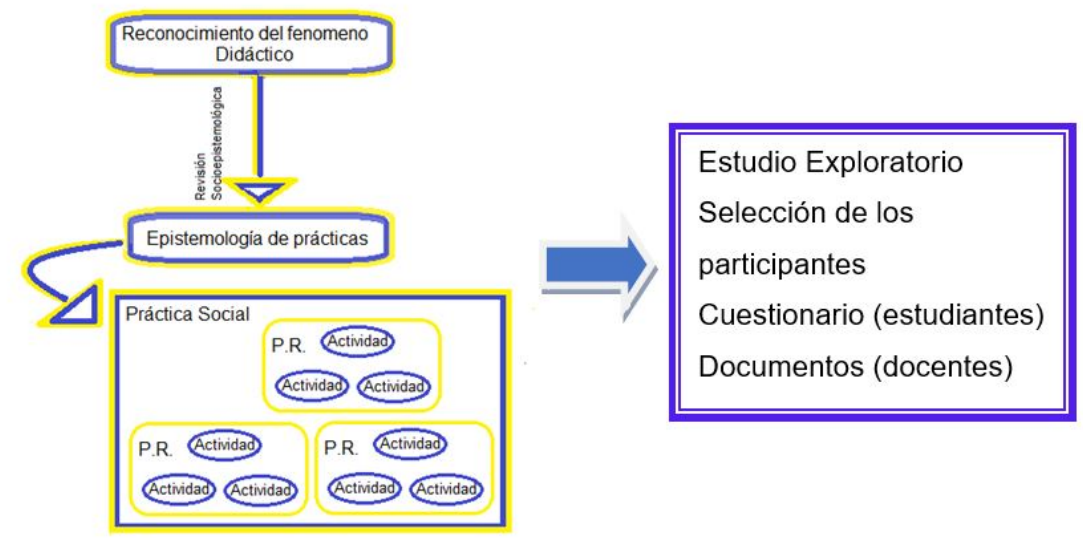

FIGURA 7

Diseño metodológico

Fuente: Elaboración propia

\section{Participantes}

En este estudio exploratorio se considera analizar dichas prácticas para estudiantes de ingeniería. En los programas de ingeniería como parte de la formación de ciencias básicas, el estudiantado cursa una clase donde el tema de inducción matemática está incluido (matemáticas discretas I). En esta investigación participaron cuatro estudiantes de ingeniería de una universidad en la frontera norte de México y dos docentes que impartían matemáticas discretas I en la misma universidad.

Criterio de selección para estudiantes. Criterio (1): estudiantes cursando matemáticas discretas I. Estudiantes deben tener el mejor desempeño académico. Criterio (2): estudiantes cursando la clase de matemáticas II, lo cual indica que en el semestre anterior vieron inducción matemática. Estudiantes deben tener el mejor desempeño académico de su grupo.

Se seleccionaron 2 estudiantes que satisfacían el criterio 1, y 2 estudiantes con el criterio 2 . El criterio de selección se ejecutó de esta manera para que las y los estudiantes pudieran contestar todas las preguntas del cuestionario que se les aplicó y evitar dejar algún problema sin respuesta o contestar con un "no sé".

Con respecto a los docentes, eran docentes activos, una de ellas impartía matemáticas discretas I al momento de que se llevó acabo esta investigación, y la otra docente impartió esa materia durante los cuatro años anteriores. El criterio de selección de los docentes fue su disponibilidad e interés en participar en la investigación.

\section{Fuentes de datos}

La fuente de datos para este estudio exploratorio fue un cuestionario. Este cuestionario fue aplicado a los 4 estudiantes. También se implementó una entrevista a un docente y se hizo la indagación en los apuntes de otro docente. Él cuestionario consiste de 3 problemas de inducción matemática en formato de pregunta abierta. En el primer problema se pidió demostrar una conjetura de una serie de sumas utilizando inducción 
matemática. En el segundo se pidió hacer una conjetura de una serie de sumas y luego demostrarla por inducción matemática, pero en este problema únicamente se les da la serie de sumas. El último es un problema donde se les pidió conjeturar un problema geométrico y para ello se les da una tabla con varios casos particulares. La Figura 8 muestra las preguntas del cuestionario.

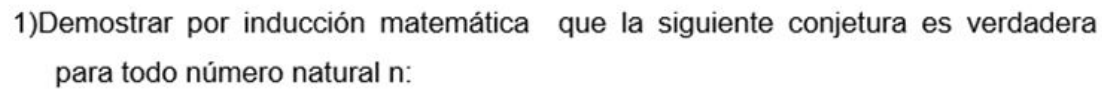

$$
1^{3}+2^{3}+3^{3}+\ldots \ldots \ldots+n^{3}=\left(\frac{n(n+1)}{2}\right)^{2}
$$

2) Formular una conjetura acerca de la suma de $n$ elementos en la serie:

$$
\begin{aligned}
& \frac{1}{1 \times 2}+\frac{1}{2 \times 3}+\frac{1}{3 \times 4}+\ldots \\
& \text { Y prueba tu conjetura con inducción matemática }
\end{aligned}
$$

3) Que numero de diagonales puede uno dibujar en un polígono (convexo) de $n$ vértices y demuéstralo con inducción matemática.

\begin{tabular}{llll}
\hline Poligono & Dibujo & No.de Vertices & No. de Diagonales \\
\hline Triángulo & & 3 & 0 \\
Cuadrado & & 3 \\
Pentagono
\end{tabular}

FIGURA 8

\section{Preguntas del cuestionario}

Fuente: Elaboración propia

Respecto a la entrevista, esta fue elaborada para explorar las dificultades didácticas de docentes cuando ven inducción matemática en su aula de clases. La entrevista consiste de las siguientes preguntas: (1) ¿Imparte usted el tema de inducción matemática en su curso de Matemáticas Discretas I? (2) ¿Cómo considera ese tema respecto a su complejidad? (3) ¿Qué tipo de ejercicios pone? (4) ¿Por qué no maneja otro tipo de ejercicios como por ejemplo de divisibilidad? (5) ¿Considera que la inducción matemática le servirá más adelante, como en otras materias o en su ejercicio profesional?

\section{ANÁLISIS DE DATOS}

Para el análisis histórico-epistemológico, se utilizaron traducciones al español de los trabajos originales. Se organizó cronológicamente en dos periodos. Luego se evaluó la evidencia de la inducción matemática. En el caso del arithmeticorum libri duo se preparó la traducción del italiano al español con la ayuda de un traductor y con la asesoría de D. Napolitani, autor del proyecto de publicaciones de las obras de Maurolico (Napolitani, 2000).

Para el análisis de los datos del alumnado, se nombraron como alumnos/as A, B, C y D. El/la alumno A y B son estudiantes de Matemáticas Discretas II, y los alumnos/as C y D son las y los estudiantes de Matemáticas Discretas I. A los 4 estudiantes se les aplicó el mismo cuestionario. Se utilizaron 3 categorías para el análisis de los datos: las dificultades cognitivas, dificultades didácticas, y dificultades epistemológicas. Respecto a los docentes se nombraron maestra 1 y maestra 2. Solo la maestra 1 fue entrevistada. La maestra 2 fue investigada 
por medio de las notas de clase de sus estudiantes. Para el análisis de los datos de las maestras, se utilizaron los temas de las dificultades didácticas identificadas previamente.

\section{Resultados}

\section{Los resultados del análisis histórico-epistemológico}

Los resultados del análisis histórico-epistemológico de la inducción matemática muestran que la práctica social asociada a la inducción matemática es la demostración como Crespo (2007) la identificó previamente. También se identificaron 2 prácticas de referencia asociadas a la inducción matemática, una es la validación de resultados debido a que en las comunidades matemáticas de los periodos investigados era la actividad que preponderaba y la otra es la reconstrucción de la matemática (griega) dándose entre los siglos XV al XVII. En ellas se encuentran actividades, que son reguladas por la práctica social demostración, esta es fundamental en la generación del significado de la inducción matemática (Crespo, 2007). La actividad que define a la práctica de referencia de la validación de resultados es el hacer matemática. En la reconstrucción de la matemática como práctica de referencia se encuentran tres actividades: analizar, interpretar y comparar.

El ser humano siempre ha tenido la necesidad de saber cómo, por qué, dónde, para qué, es decir, explicar el sentido y la naturaleza de todo. El humano buscó respuestas en argumentos místicos, nada racionales, pero con la evolución de la humanidad, llegó el momento en que una cultura trató de buscar otro tipo de respuestas, la cultura griega, está buscó soluciones racionales, este proceso fue dándose lentamente debido a la mitología arraigada en cada cultura. Entonces así fue como los griegos introdujeron el método deductivo. Los griegos tuvieron la necesidad de saber, si los resultados hechos por civilizaciones anteriores eran correctos o eran contradictorios de ahí la necesidad de demostrar ciertos legados para dar validez a sus resultados. Esta necesidad da lugar a la práctica social de la demostración.

Esta investigación considera que la esencia de la matemática radica en el tipo de justificaciones que tiene, en las argumentaciones que da, es decir, en sus demostraciones. Desde la antigüedad los matemáticos tenían la obligación de demostrar las matemáticas que hacían, estas demostraciones fueron meramente deductivas.

La práctica social de la demostración, no es la misma de una comunidad a otra, se ha modificado y evolucionado de una cultura a otra; no es la misma para distintas comunidades matemáticas, pero su finalidad básica ha sido la legitimación del saber matemático, aunque no es esta su única función (Crespo, 2007). La demostración debe unirse a la normativa, es la que establece cuáles serán las demostraciones aceptadas por esa comunidad. La normativa es distinta, pero la demostración es, para todas ellas, una práctica social tendiente a la validación de resultados (Crespo, 2007).

Desde la antigüedad hasta estos días, todo teorema, principio o proposición matemática es demostrada para su validez. Para ello se utilizan métodos o procedimientos matemáticos, uno de esos métodos es la inducción matemática. Por lo cual la práctica social asociada a su generación es la demostración, como se ha fundamentado en este estudio socioepistemológico.

La demostración como práctica social regula a las prácticas de referencia y a sus actividades. El hacer matemática es la actividad asociada a la práctica de referencia de la validación de resultados. Esta actividad se refiere a desarrollar actividades matemáticas, como investigar matemática, enseñar matemática. Durante el estudio histórico-epistemológico se observó cómo trabajos tan importantes como el de Euclides utilizan métodos para demostrar la matemática, así como también trabajos de las diferentes comunidades matemáticas griegas, hindús y árabes. Estas comunidades hicieron matemática la cual fue validada al demostrar sus resultados y argumentos. Así es como esta investigación reconoce la construcción social de la inducción matemática. 
En arithmeticorum libri duo de Maurolico se demostraron propiedades y proposiciones acerca de sistemas de numeración (Napolitani, 2000). Para ello, Maurolico utilizó procedimientos los cuales lo llevaron a usar una u otra proposición. Necesitó tener la certeza de que lo que hacía era correcto y válido y fue entonces que empleó la inducción matemática para demostrar (Napolitani, 2000). Al igual en el triángulo aritmético de Pascal (1995) se utilizaron los componentes del triángulo y se elaboraron proposiciones, siendo en la consecuencia XII donde se demostró algo que tendía al infinito. Lo demostró con la inducción matemática para fundamentar y satisfacer la necesidad de justificar su trabajo.

En la construcción social de la inducción matemática se identificó a la reconstrucción de la matemática como otra práctica de referencia con las actividades de analizar, interpretar y comparar. Estas actividades fueron fundamentales en el pensamiento de estos matemáticos para poder lograr hacer sus reconstrucciones matemáticas, así como sus trabajos originales de los cuales se generó la inducción matemática.

\section{Resultados del estudio exploratorio}

En este estudio exploratorio de 4 estudiantes de ingeniería, se reconocieron las dificultades cognitivas, didácticas y epistemológicas con las que las y los estudiantes se encuentran en el proceso de aprendizaje de la inducción matemática previamente identificadas en los antecedentes. El cuestionario cuenta con un problema con el que estuvieran acostumbrados a resolver en clase, sin que sea demasiado sencillo o típico (problema 1). Por ello con este problema se esperaba que la mayoría de las y los estudiantes pudieran resolverlo correctamente, y de los 4 estudiantes, 2 lo resolvieron correctamente sin problema.

El problema 2 es de una serie de sumas igual que el primer problema. Sin embargo, aquí el estudiante primero tenía que conjeturar y luego demostrarlo con la inducción matemática. El hecho de impartir en la clase un solo tipo de ejercicios de inducción matemática no significa que las y los estudiantes asimilan totalmente ese tipo. Si el o la estudiante no asimila y comprende la inducción matemática no se da un aprendizaje significativo, aunque se aborde un solo tipo o varios de inducción matemática como se evidenció. La falta de costumbre para conjeturar, así como para resolver problemas geométricos con inducción matemática se identificó en el problema 3 del cuestionario.

En los resultados encontrados del estudio exploratorio con las y los estudiantes se pudo identificar que los tres tipos de dificultades en torno al aprendizaje y enseñanza de la inducción matemática estuvieron presentes en los 4 estudiantes. Las y los estudiantes $\mathrm{C}$ y D son los que vieron recientemente la inducción matemática, sin embargo, fueron los que presentaron más dificultades cognitivas.

Respecto a las dificultades cognitivas, los 4 estudiantes mostraron dificultad para conjeturar, poco dominio y familiaridad con problemas geométricos de inducción matemática. Los alumnos/as B, C y D presentaron una pobre manipulación algebraica. Además, el alumno D mostró una carencia conceptual en el aspecto algebraico. Deficiencias conceptuales acerca del significado de la inducción matemática fueron identificadas en los alumnos/as $\mathrm{C}$ y $\mathrm{D}$.

Las dificultades didácticas que se apreciaron de los datos del cuestionario de las y los estudiantes indican que el profesorado no incluye problemas donde el estudiante necesite conjeturar. Además, el alumnado no fue expuesto a diferentes tipos de ejercicios incluyendo problemas geométricos de inducción matemática. Esto repercute el estudiantado por utilizar un solo tipo de notación y confundirse si se les presenta otra notación. De acuerdo a Avital y Libeskind (1978) esto sucede por la falta de dominio del profesorado.

Estudiantes presentaron dificultades epistemológicas relacionadas con el formalismo y rigor que se maneja en la inducción matemática. La dificultad de utilizar notaciones muy rigorosas fue identificada. Además, el alumno B mostró dificultad para comunicar sus resultados.

El estudiantado no pudo resolver los problemas del cuestionario. Ellos no pudieron conjeturar para llegar a la demostración. Del análisis de sus respuestas, podemos decir que un estudiante de ingeniería busca algoritmos, patrones, y practicidad para tratar de resolver los problemas. Estos aspectos no son parte de la 
inducción matemática. Lo que hace que la práctica social, la demostración, no sea compartida por las y los estudiantes de ingeniería. Por lo cual identificar elementos útiles para un rediseño del discurso escolar de la inducción matemática no fue posible.

Los resultados del análisis de los datos de los docentes corroboraron las dificultades didácticas mencionadas en los antecedentes. Las respuestas de la maestra 1 en la entrevista elaborada mostraron cómo la falta de dominio, mal manejo de ejercicios y poca variedad de ejercicios conduce a que las y los estudiantes crean que la inducción matemática es exclusivamente para serie de sumas. Esto los deja desarmados para demostrar otro tipo de problemas como se ejemplificó en los problemas aplicados a las y los estudiantes participantes, en el problema geométrico, donde no cabe duda que no tienen el dominio necesario para resolverlo.

La maestra 1 reconoce la complejidad de la inducción matemática. Ella mencionó "La verdad sí es muy complicado pero trato de poner ejercicios sencillos" (Maestra 1, comunicación personal, 10 de septiembre de 2010). A pesar del intento de la maestra de tratar de poner ejercicios sencillos, se cae en el error de únicamente utilizar ejercicios de series de sumas. La maestra 1 indicó que imparte ejercicios "varios de series de sumas" (Maestra 1, comunicación personal, 10 de septiembre de 2010). Se le cuestionó por qué no abordó otro tipo de ejercicios, por ejemplo de divisibilidad. Ella respondió que "la verdad es que es un tema muy complejo, y si con los ejercicios de sumas batallan las y los estudiantes, ahora con problemas de divisibilidad no la hacen, en verdad no pueden" (Maestra 1, comunicación personal, 10 de septiembre de 2010). Se observó como las y los estudiantes están expuestos a ejercicios de series de sumas cuando se imparte la inducción matemática.

Se habló también acerca de la utilidad de la inducción matemática para las y los estudiantes de ingeniería. La maestra 1 mencionó que la utilidad para las y los estudiantes "en otras materias y su ejercicio profesional no sé, pero en mi clase si porque ellos pueden comprobar los resultados que les arroje un programa de computación acerca de alguna serie de sumas, si lo comprueban en su cuaderno" (Maestra 1, comunicación personal, 10 de septiembre de 2010). Se observó cómo se centra únicamente en la serie de sumas y como ella misma no tiene claro que tan útil es este tema para este tipo de estudiantes.

Los documentos analizados de la maestra 2 fueron las notas de clase de tres alumnos/as escogidos/as aleatoriamente, a los/las cuales se les pidió su cuaderno de la materia de matemáticas discretas I. Esto para hacer una exploración de cómo se abordó el tema de inducción matemática. Lo que se encontró es que las palabras de inducción matemática únicamente se encontraron en el temario que tienen en el inicio de su cuaderno. En los apuntes de los tres estudiantes de la misma clase, no se encontró ni un solo problema o ejercicio de inducción matemática. Lo cual lleva a deducir que la maestra 2 no imparte inducción matemática. Esta acción puede ocurrir por varias razones una de ellas es la falta de dominio o porque la considera muy compleja. Por falta de tiempo no puede ser porque es un tema que se aborda en la primera unidad, y regularmente los temas que por falta de tiempo no se cubren en clase son los últimos. Por lo tanto, las primeras dos razones son las más factibles, estas dificultades fueron identificadas también en la revisión bibliográfica.

De manera resumida los resultados de los docentes, incluyen las siguientes dificultades previamente identificadas en los antecedentes: (a) la falta de dominio; (b) mal manejo de ejercicios; y (c) una limitada variedad de ejercicios lo que puede conducir a que las y los estudiantes crean que la inducción matemática es exclusivamente para serie de sumas.

Después de analizar todos los datos, se puede concluir que evidentemente la inducción matemática está asociada a un fenómeno didáctico el cual afecta tanto a estudiantes como a docentes a nivel superior. Se evidencia claramente la problemática existente en torno el aprendizaje y enseñanza de la inducción matemática. Esta problemática coincide con la mencionada previamente en la revisión bibliográfica. 


\section{CONCLUSIONES Y DISCUSIÓN}

Esta investigación se desarrolló desde un enfoque social, es por ello que se trabajó con la socioepistemología; se considera que toda actividad matemática es meramente humana y por lo cual no se puede deslindar el aspecto social de la creación o generación de un objeto matemático así como tampoco de su proceso de enseñanzaaprendizaje y del factor social.

La revisión socioepistemológica llevada a cabo fue con el propósito de entender cómo se generó la inducción matemática, y para ello se hizo una revisión exhaustiva de varios materiales, obras originales de algunos de los autores, se tuvo el apoyo de traductores y se analizaron traducciones importantes de partes de las obras de los personajes investigados.

Esta investigación muestra la manera en que emergió, en que surgió y cómo se generó la inducción matemática tomando en cuenta el contexto histórico por el que se atravesaba en esos momentos. Por lo cual, se concluye que la inducción matemática surgió por la razón de querer demostrar ciertas proposiciones, ciertos resultados, algunos de esos resultados eran considerados verdaderos y con la inducción matemática se comprobaba que se estaba en lo cierto, otros se construyeron y validaron gracias a la inducción matemática. Con esto se reafirma el hecho de que las matemáticas basan su esencia en las demostraciones, hecho que se pudo observar a lo largo de este trabajo de investigación.

Después de la revisión socioepistemológica y la epistemología de prácticas de carácter histórico se ha llegado a la siguiente conclusión: la inducción matemática es generada por la práctica social de la demostración, práctica que fue identificada en su construcción de origen y que regula la validación de resultados, así como la reconstrucción de la matemática.

Se agrega a esta discusión, la socioepistemología no estudia nada más el cómo enseñar sino que cuestiona el qué enseñar, y este trabajo es una evidencia de ello, porque con él se puede discutir si es factible o no que la inducción matemática siga en las aulas de los institutos de ingeniería. Por lo tanto, se puede concluir que el alumnado de ingeniería no comparte el interés de esta práctica, es decir no siente o no tiene la necesidad o motivación para demostrar y la inducción matemática surgió para ello precisamente. Además, el estudiantado de ingeniería busca la practicidad y los algoritmos, aspectos que en la inducción matemática no están contenidos.

En el estudio exploratorio de los 4 estudiantes se corroboraron las dificultades identificadas en la revisión bibliográfica. El alumnado no pudo conjeturar para llegar a la demostración. Además, no están acostumbrados a resolver problemas geométricos con inducción matemática. Quienes participaron tuvieron una pobre manipulación algebraica y manifiestan deficiencias conceptuales acerca del significado de la inducción matemática. De la misma forma se identificaron dificultades didácticas y epistemológicas en las respuestas del cuestionario. Los resultados de la exploración a los docentes mostraron una falta de dominio de la inducción matemática, un mal uso de ejercicios y ejemplos de este tema lo que ha contribuido a confusiones y malas concepciones en las y los estudiantes. A pesar de que esta investigación se ejecutó únicamente con cuatro estudiantes y dos maestras, los resultados ayudan a entender lo que pasa en las clases de matemáticas de ingeniería cuando se aborda la inducción matemática. Al mismo tiempo se ratifica que este fenómeno didáctico asociado a la inducción matemática previamente identificado en la revisión bibliográfica existe a nivel universitario.

La inducción matemática es un método de demostración importante en las matemáticas, pero debido a la práctica social asociada a su génesis en este trabajo se presentan elementos que indican que una resignificación es muy compleja e inapropiada para llevarla al aula de estudiantes de ingeniería. Después del análisis históricoepistemológico, se puede reflexionar acerca de la pertinencia de abordar la inducción matemática en las clases de ingeniería. La clase de matemáticas discretas I la cursan todos las y los estudiantes de ingeniería. Los programas de ingeniería de la Universidad donde se desarrolló este trabajo son: industrial, civil, manufactura, sistemas digitales, sistemas computacionales, eléctrica, mecatrónica, entre otras de recién incorporación 
como biomédica, sistemas automotrices y nanotecnología. Es relevante analizar que competencias puede ofrecer la inducción matemática a un estudiante de cada particular ingeniería.

De manera general, la inducción matemática puede ayudar a desarrollar un razonamiento inductivo que es parte de una formación de ingeniero y su práctica profesional. Sin embargo, este tipo de razonamiento también puede ser desarrollado por medio de otros tópicos de matemáticas. Otro beneficio de utilizar la inducción matemática para futuros ingenieros, como este método de demostración requiere una manipulación algebraica, la cual ayudaría al estudiantado a mejorar su manipulación algebraica que es necesaria para su formación. Sin embargo, las habilidades para tener una buena manipulación algebraica, se puede lograr de diferentes maneras sin tener que abordar la inducción matemática. Por ello, el implementar alguna situación de aprendizaje no es conveniente, porque aunque se trate de arribar al tema, de una forma o de otra, el/la estudiante no logra entender inducción matemática y continúa con sus mismos razonamientos erróneos, esto debido a que la práctica social es diferente a las necesidades y motivaciones de las y los estudiantes de ingeniería.

En ningún momento de esta investigación se afirma que la inducción matemática no sirve para el alumnado de ingeniería o que no le va a ser de ayuda en su ejercicio profesional, eso no se concluye en esta investigación, sino lo que se quiere comunicar es simplemente que el estudiantado no tiene la motivación necesaria para querer validar un resultado o proposición, la persona ingeniera busca patrones, busca seguir un procedimiento, un algoritmo y ponerlo en práctica, no demostrarlo. Sin embargo, si se reflexiona en una ingeniería particular y se decide que la inducción matemática es indispensable en la formación de profesionales en ingeniería, entonces se tendrán que buscar y dar las condiciones para que se plantee una situación de aprendizaje en el aula que proporcione la transposición didáctica de la inducción matemática a contextos y situaciones que sean entendidos por este estudiantado para que reconozcan su utilidad y les aumente su motivación por aprenderla.

\section{REFERENCIAS BIBLIOGRÁFICAS}

Andrew, L. (2007). Reasons why students have difficulties with mathematical induction (Report). Greeley: University of Northern Colorado.

Avital, S. y Libeskind, S. (1978). Mathematical Induction in the classroom: Didactical and Mathematical Issues. Educational Studies in Mathematics, 9(4), 429-438.

Buendía, G. (2004). Una epistemología del aspecto periódico de las funciones en un marco de prácticas sociales (Un estudio socioepistemológico). (Tesis de doctorado). Centro de Investigación y de Estudios Avanzados del Instituto Politécnico Nacional, México.

Buendía, G. (2006). Una socioepistemología del aspecto periódico de las funciones. Revista Latinoamericana de Investigación en Matemática Educativa, 9(2), 227-251.

Buendía, G. y Cordero, F. (2005). Prediction in the periodic aspect as generators of knowledge in a social practice framework. A siocioepistemological study. Educational Studies in Mathematics, 58(3), 299-333.

Bussey, W. (1917). The Origin of Mathematical Induction. The American Mathematical Monthly, 24(5), 199-207.

Cajori, F. (1909). Shorter Notices (rewiew Ueber das Wesen der Mathematik), Bulletin of the American Mathematical Society, 15(8), 405-409.

Cajori, F. (1918). Origin of the name "Mathematical Induction". The American Mathematical Monthly, 25(5), 197-201.

Castro, E., Cañadas, M. y Molina, M. (2010). El razonamiento inductivo como generador de conocimiento matemático. UNO, 54, 55-67.

Covián, O. (2005). El papel del conocimiento matemático en la construcción de la vivienda tradicional. El caso de la cultura maya (Tesis de maestría no publicada). Centro de Investigación y de Estudios Avanzados del Instituto Politécnico Nacional, México. 
María De los Ángeles Cruz Quiñones, et al. Caracterización de las prácticas sociales asociadas a l...

Crespo, C. (2007). Las argumentaciones matemáticas desde la visión de la socioepistemología (Tesis de doctorado no publicada). Centro de Investigación en Ciencia Aplicada y Tecnología Avanzada del Instituto Politécnico Nacional, México.

Cusi, A. y Malara, N. (2008). Improving awareness about the meaning of the principle of mathematical induction. En O. Figueras, J. Cortina, S. Alatorre, T. Rojano y A. Sepúlveda (Eds.). Proceedings of the Joint Meeting of PME 32 and PME-NA (pp. 393-398). Morelia, México: Cinvestav-UMSNH

Ernest, P. (1982). Mathematical Induction: A Recurring Theme. The Mathematical Gazette, 66(436), 120-125.

Ernest, P. (1984). Mathematical Induction: A Pedagogical Discussion. Educational Studies in Mathematics, 15(2), 173-189.

Euclides (1991). Elementos, (Puertas Castaños, M. Trad.). España: Gredos.

Mason, J. y Spence, M. (1999). Beyond mere knowledge of mathematics: The importance of knowing-to act in the moment. Educational Studies in Mathematics, 38(1), 135-161.

Montiel, G. (2005). Estudio Socioepistemológico de la Función Trigonométrica (Tesis de doctorado no publicada). Centro de Investigación en Ciencia Aplicada y Tecnología Avanzada, México.

Movshovitz, N. (1993). The False Coin Problem, Mathematical Induction, and Knowledge Fragility. The Journal of Mathematical Behavior, 12(3), 253-268.

Napolitani, D. (2000). Arithmeticorum libri duo. Francisci Maurolyci Mathematica. Recuperado de http://mauroli co.free.fr/edizioni/arithmet/ariduo/intro.htm

Pascal, B. (1995). Obras Matemáticas. México, D.F.: Mathema.

Ron, G. y Dreyfus, T. (2004). The use of models in teaching proof by mathematical induction. En M. J. Hoines y A. B. Fuglestad (Eds.), Proceedings of the twenty-eighth conference of the international group for the Psychology of Mathematics education PME (pp.113-120). Bergen, Norge: Bergen University College.

Sampieri, R. (2006). Metodologia de la investigación. México: Mc Graw Hill.

Tchoshanov, M., Cruz, M., Huereca, K., Shakirova, K., Shakirova, L. e Ibragimova, E. (2017). Examination of Lower Secondary Mathematics Teachers' Content Knowledge and Its Connection to Students' Performance. International Journal of Science and Mathematics Education, 15(4), 683-702.

\section{BY-NC-ND}

